\title{
ORIGINAL
}

\section{BOLETÍN EPIDEMIOLÓGICO DE LA COMUNIDAD DE MADRID: ENCUESTA SOBRE SU DIFUSIÓN y PERCEPCIÓN ENTRE LOS MÉDICOS DE ATENCIÓN PRIMARIA EN EL AÑO 2000}

\author{
Silvia Fernández Rodríguez, Belén Zorrilla Torras, Rosa Ramírez Fernández, M." Carmen \\ Álvarez Castillo, Dulce López-Gay Lucio, Cosuelo Ibáñez Martín y Rafael Bueno Vallejos \\ Servicio de Epidemiología. Instituto de Salud Pública. Consejería de Sanidad. Comunidad de Madrid.

\section{RESUMEN} \\ Fundamento:. El objetivo es conocer la difusión y percep- \\ ción del Boletín Epidemiológico de la Comunidad de Madrid \\ (BECAM) entre los médicos de atención primaria, con el fin de \\ adaptar la publicación al interés de sus lectores. \\ Método: Encuesta telefónica entre los médicos de primaria \\ de la Comunidad de Madrid, preguntando por la frecuencia de

\section{ABSTRACT} \\ The Autonomous Community of Madrid \\ Epidemiological Bulletin. A Survey \\ Regarding its Dissemination and \\ Opinion Thereof on Among Primary \\ Care Physicians for the Year 2000
} lectura, interés y utilidad de la información contenida El tamaño muestral se estimó en 346 médicos. Se realizó un muestreo bietápico por conglomerados en la primera etapa, con selección aleatoria de 125 centros de salud y 2,7 médicos por centro, siendo el $17 \%$ coordinadores de equipo. Se comparan los resultados entre médicos y coordinadores mediante Chi-cuadrado y test exacto de Fisher, con Epi-Info v.6.

Resultados: Se realizó un total de 305 entrevistas: 245 médicos y 60 coordinadores. Sabían de la existencia del BECAM el $91,5 \%$ (IC 95\%: 88,1-94,8), y el 27,2\% (IC 95\%: 21,9-32,5) conocía más del $50 \%$ de los números editados durante el año. El 92,4\% (IC 95\%: 89,4-95,8) lo consideraba interesante o muy interesante, puntuando con una media de 3,5 sobre 5 su utilidad. De las secciones fijas, la mejor valorada es Brotes Epidémicos, y de los informes, los relacionados con enfermedad meningocócia, tuberculosis y VIH/Sida.

Conclusiones: El BECAM es una publicación que, aunque no es conocida ampliamente entre los médicos de primaria de nuestra Comunidad, cuando se lee es bien valorada; siendo así un instrumento útil de retroalimentación en la Red de Vigilancia Epidemiológica.

Palabras clave: Vigilancia epidemiológica. Revistas científicas. Encuesta.

Correspondencia:

Silvia Fernández Rodríguez

Servicio de Epidemiología

Instituto de Salud Pública de la Comunidad de Madrid

Consejería de Sanidad. Comunidad de Madrid

C/ Aduana 29, 1. ${ }^{\text {a }}$ planta.

28013-Madrid. Teléfono: 91.5867256. Fax: 91.5867689.

Correspondencia: silvia.fernandez@madrid.org
Background: The Autonomous Community of Madrid Epidemiological Bulletin is the main communications link between epidemiological monitoring system and health care professionals. The purpose of this study is that of ascertaining the dissemination and opinion of this Autonomous Community of Madrid Epidemiological Bulletin among primary care physicians for the purpose of adapting this publication to its readers' interests.

Method: A telephone survey among primary care physicians in the Autonomous Community of Madrid, asking how often they read the Bulletin, the interest and usefulness of the information included in it. The sample size was estimated at 346 physicians. A two-stage sampling process was carried out by cluster sampling in the first stage, randomly selecting 125 health care centers and 2.7 physicians per center, $17 \%$ being primary care team coordinators. A comparison is made of the results among physicians and coordinators by means of the Chi-square and Fisher's Exact Test method, with Epi-Info v.6.

Results: A total of 305 surveys were conducted (245 physicians and 60 coordinators). There was an awareness of the existence of the Autonomous Community of Madrid Epidemiological Bulletin on the part of $91.5 \%$ (CI 95\%: 88.1-94.8), and 27.2\% (CI $95 \%: 21.9-32.5$ ) were familiar with more than $50 \%$ of the last issues published. A total of $92.4 \%$ (CI 95\%: 89.4-95.8) considered the Bulletin to be interesting or highly interesting, grading its usefulness an average of 3.5 on a maximum scale of 5 . Of the permanent sections, the most highly-valued was Epidemic Outbreaks, those reports related to meningococcal infection, tuberculosis and HIV/AIDS being the most highly-valued.

Conclusions: The Autonomous Community of Madrid Epidemiological Bulletin is a publication which, although not widely-known by the primary care physicians in the Community, is well-valued when it is read, thus being a useful feedback tool within the Epidemiological Monitoring System.

Keywords: Epidemiological monitoring. Scientific journal. Survey. 


\section{INTRODUCCIÓN}

El Boletín Epidemiológico de la Comunidad de Madrid (BECAM) es el principal instrumento de comunicación establecido entre la vigilancia epidemiológica y los profesionales sanitarios. Su propósito es hacer llegar la información agregada a nivel regional, generada a partir de los diferentes sistemas de vigilancia; en especial a las redes asistenciales cuyos profesionales, como notificadores, constituyen el soporte de la mayor parte de los sistemas de vigilancia.

La preocupación desde Salud Pública siempre ha sido conseguir que este Boletín cumpliese su verdadera razón de ser, llegando a todos aquellos profesionales de nuestra región, y resultándoles útil para el desempeño de su labor asistencial. Así, en el año 1993 se llevó a cabo un estudio cualitativo sobre el mismo entre médicos de atención primaria, cuyo resultado mostró que sólo lo valoraban en función de la devolución de información de los sistemas basados en la notificación de casos. (Documento Interno: Actitudes de los profesionales sanitarios y de la población frente a la vigilancia epidemiológica en la Comunidad de Madrid; 1993).

Desde entonces, el BECAM ha experimentado cambios en su presentación, diseño, y especialmente en su contenido, intentando satisfacer las expectativas de los profesionales a los que va dirigido y en paralelo al desarrollo de la Salud Pública. En este sentido, se han incorporado informes relacionados con nuevos sistemas de vigilancia para las enfermedades transmisibles ( Red de Médicos Centinela, encuestas de serovigilancia y de prevalencia de infección tuberculosa,...); y se ha ampliado la información a las enfermedades crónicas y sus factores de riesgo, fundamentalmente de aquellos relacionados con los estilos de vida y enfermedades emergentes, como los trastornos del comportamiento alimentario.
Producto de esa transformación y preocupación por conseguir que el BECAM sea no sólo un instrumento útil de devolución de información epidemiológica, sino también una herramienta de actualización de conocimientos, se diseñó un cuestionario dirigido a los médicos de Atención Primaria de la Comunidad de Madrid, puesto que las encuestas de opinión son uno de los procedimientos más apropiados para poder conocer el impacto de una publicación entre sus lectores ${ }^{1}$. El objetivo de la misma era evaluar el impacto de esta revista en relación a su distribución, así como el interés que despertaba entre estos profesionales su contenido actual. También se les solicitó sugerencias sobre otros temas de su interés que no apareciesen reflejados todavía en el BECAM.

\section{MATERIAL Y MÉTODOS}

A finales del año 2000 se llevó a cabo una encuesta telefónica entre los médicos de atención primaria de nuestra Comunidad. El tamaño de la muestra se estimó en 346 médicos para un universo muestral de 3.413 médicos, asumiendo una proporción de lectores del Boletín del 50\%, una precisión relativa del $10 \%$ y un nivel de confianza del 95\%. El marco muestral es el registro de notificadores al Sistema de Enfermedades de Declaración Obligatoria de la Comunidad de Madrid; en el que figuran 3.413 médicos de atención primaria distribuidos en 290 centros de salud.

Para el cálculo del tamaño muestral se ha utilizado Epidat v.2. Se realizó un muestreo bietápico con conglomerados en la primera etapa; las unidades de primera etapa estaban constituidas por los centros de salud, y las unidades de segunda etapa por los médicos (generalistas o pediatras) adscritos al centro.

Se seleccionaron aleatoriamente 125 centros de salud (43,1\% del total de centros). Una vez seleccionados los centros, y teniendo en cuenta el criterio de economía y con- 
veniencia en la recogida de información, se seleccionó una media de 2,7 médicos por centro de salud, de los que el $17 \%$ eran coordinadores de equipo. Al contactar telefónicamente con el centro, se solicitaba que se pusiesen al teléfono o bien los médicos que más cerca se encontraban de la recepción en ese momento, o bien aquellos que estuviesen disponibles.

El error estándar de los estimadores se ha calculado mediante la aproximación Desviación Linearizada de Taylor, a través el módulo CSample de Epi-Info v.6. Con el fin de identificar posibles diferencias entre médicos y coordinadores de equipo, se compararon los resultados obtenidos entre ambos mediante los test de Chi-cuadrado y exacto de Fisher de comparación de proporciones, utilizando el programa informático Epi-Info v.6.

Las encuesta contenía preguntas referentes a si conocían o no el BECAM; a los que no estaban seguros en la respuesta, se les aclaraba que se trataba de una publicación bimensual, que había cambiado el color de sus tapas de gris a morado en el año anterior. También se investigaba con qué frecuencia lo leían, cómo calificaban la información contenida y la utilidad para su trabajo. Seguidamente se preguntaba por la valoración que les merecía cada uno de los apartados fijos del Boletín (EDO, Brotes epidémicos, Sistema de vigilancia microbiológica, y Red de médicos centinela); así como si había leído los informes regionales publicados en los últimos 12 meses: EDO 1998, Meningitis 1998, Hábitos de salud en adultos 1998, Sistema de vigilancia microbiológica; Vigilancia del VIH/SIDA a 30 de marzo de 1999, Temporada de gripe 1998-1999. Leishmaniosis humana 19911996, y Hábitos de salud en la población juvenil de la Comunidad de Madrid, 1999. Para finalizar, se les pedía que propusiesen 5 temas de su interés que no formaran parte de la información publicada habitualmente en él (anexo 1).

\section{RESULTADOS}

Se contactó telefónicamente con 347 médicos, siendo la tasa de no respuesta del $12,1 \%$, realizándose por tanto un total de 305 entrevistas: 245 médicos $(80,3 \%)$ y 60 coordinadores de equipo $(19,7 \%)$.

Al comparar las respuestas a las diferentes preguntas del cuestionario entre coordinadores de equipo y médicos, no se encontraron diferencias significativas, por lo que a continuación se presentan los resultados encontrados sin diferenciar por puesto de trabajo.

\section{Distribución}

Cuando se les preguntaba si conocían el BECAM, en principio respondieron afirmativamente 271 médicos $(88,8 \%$; IC 95\%:85,1-92,6); y al aclarar del tipo de revista que se trataba pasaron a ser 279 $(91,5 \%$, IC 95\% 88,1-94,8). De todos ellos 83 (27,2\%, IC 95\%: 21,9-32,5) había visto más de la mitad de los 6 números editados durante el año.

Dado que la suscripción a este Boletín es gratuita, y se puede recibir tanto en el centro de trabajo como en el domicilio particular, se les preguntó si conocían la posibilidad de inscribirse en la base de datos para la distribución del mismo de manera nominal, y 122 (40\%, IC 95\%: 34,2-45,8) respondieron afirmativamente.

En cuanto al lugar en el que cada uno de los encuestados tiene acceso al BECAM, 116 médicos (38,03\%, IC 95\%: 32,5-43,6) lo hacía en la biblioteca de su centro de trabajo; $111(36,4 \%$, IC 95\%: 31,1-41,7) en su mesa de consulta, $41(13,4 \%$, IC 95\%: $9,6-17,2)$ en su domicilio particular, y el resto $(3,6 \%$, IC $95 \%: 1,5-5,6)$ en otros lugares. Además 7 médicos recibían el BECAM en más de un lugar. 


\section{Alcance e interés \\ Valoración global}

El 29,8\% (IC 95\%: 24,5-35,2) de los 279 médicos que conocían el Boletín lo leía habitualmente (figura 1) y entre aquellos que nunca lo habían leído un 50\% (IC 95\%: 6,1-83,8), el motivo referido era la falta de tiempo.
El 92,6\% (IC 95\%: 89,4-95,8) de los lectores consideran la información publicada como interesante o muy interesante. En cuanto a la utilidad que tiene la misma para su trabajo — puntuada de 1(ninguna) a 5 (mucha) - la puntuación media fue de 3,5: el 38,4\% (IC 95\%: 32,8-44,1) dio el valor 3 $\mathrm{y}$ el $37,4 \%$ (IC 95\%: 31,5-43,2) un 4 (figura 2).

\section{Figura 1}

Frecuencia de lectura del Boletín Epidemiológico de la Comunidad de Madrid

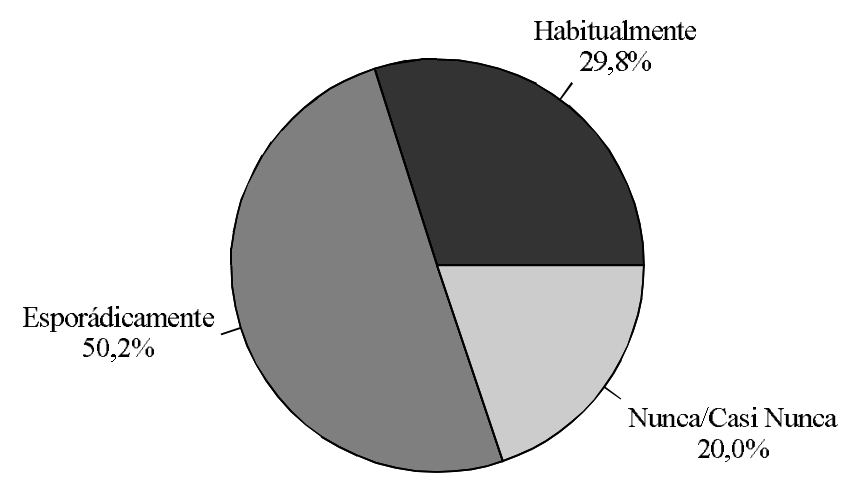

Figura 2

Utilidad de la Información publicada en el Boletín Epidemiológico de la Comunidad de Madrid para su trabajo N. ${ }^{\circ}$

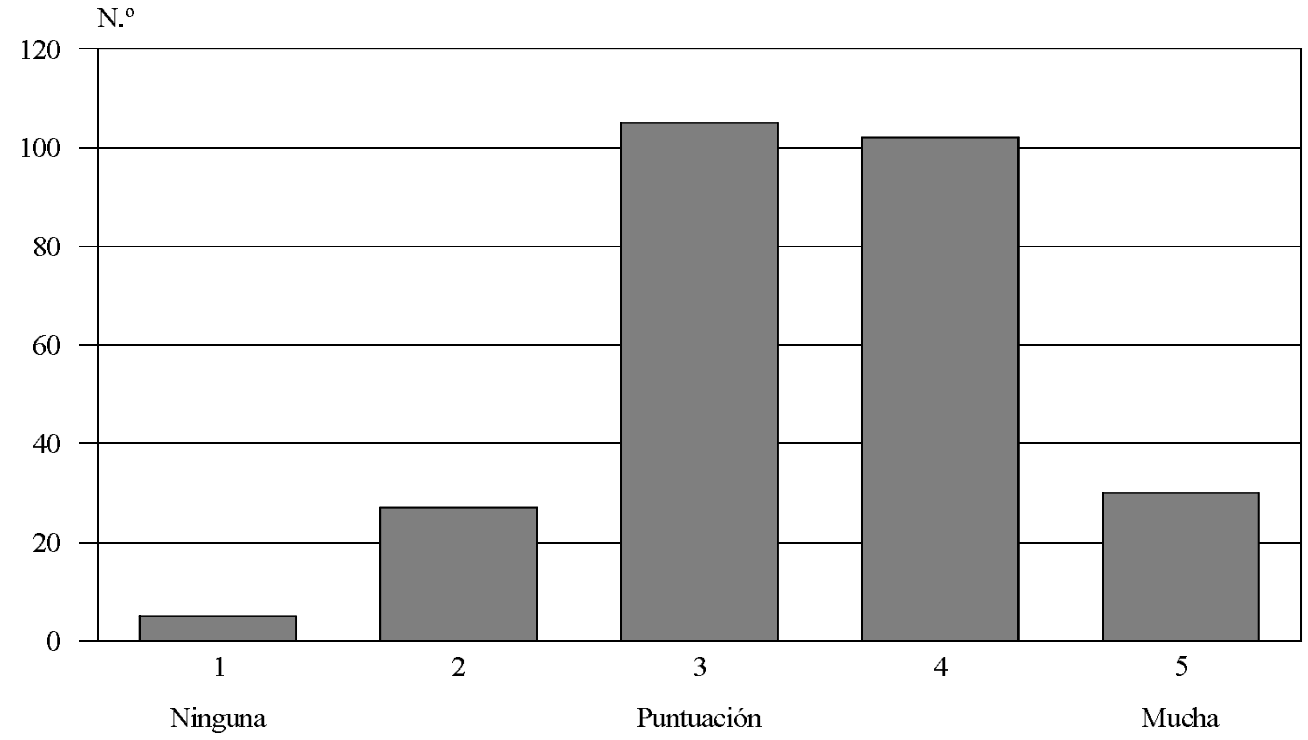




\section{Valoración de las Secciones fijas}

Ante la pregunta sobre el interés que concedían a cada una de las secciones fijas que aparecen en la publicación, referida a la información bimensual sobre Enfermedades de Declaración Obligatoria, Brotes y Red de Médicos Centinela; y trimestral sobre el Sistema de Notificación Microbiológica, resultó ser la referente a Brotes Epidémicos la Sección mejor valorada. Si bien todas ellas son consideradas al menos por el $71,7 \%$ (IC 95\%: 66,5-77,0) de los lectores como interesantes o muy interesantes (tabla 1).

Tabla 1

Nivel de Interés de las Secciones Fijas del Boletín Epidemiológico de la Comunidad de Madrid

\begin{tabular}{|lrrrrrrrrr|}
\hline & \multicolumn{1}{c}{ E.D.O. } & \multicolumn{2}{c}{ Brotes } & \multicolumn{2}{c|}{ R.M.C. } & \multicolumn{3}{c|}{ S.N.M. } \\
\hline & $N$ & $\%$ & \multicolumn{1}{c}{$N$} & $\%$ & \multicolumn{1}{c}{$N$} & $\%$ & $N$ & $\%$ \\
\hline Muy interesante & 74 & 27,1 & 141 & 51,6 & 60 & 22,0 & 52 & 19,0 \\
Interesante & 155 & 56,8 & 121 & 44,3 & 148 & 54,2 & 144 & 52,7 \\
Poco interesante & 42 & 15,4 & 10 & 3,7 & 56 & 20,5 & 69 & 25,3 \\
Nada interesante & 2 & 0,7 & 1 & 0,4 & 9 & 3,3 & 8 & 2,9 \\
\hline Total & 273 & 100,0 & 273 & 100,0 & 273 & 100,0 & 273 & 100,0 \\
\hline
\end{tabular}

E.D.O.= Enfermedades de Declaración Obligatoria; R.M.C. $=$ Red de Médicos Centinela; S.N.M.= Sistema de Notificación Microbiológica.

\section{Valoración de los Informes Regionales}

Con relación a los informes regionales publicados en los últimos 12 meses (tabla 2), los que más interés han suscitado han sido el Impacto de la vacunación frente a la enfermedad meningocócica, el Registro de tuberculosis y la Vigilancia del VIH/SIDA. Cabe destacar que, entre los profesionales que han leído cada uno de los informes, más del $90 \%$ los considera interesantes (tabla 2).

\section{Sugerencias}

Ante la solicitud de que enumerasen cinco temas de su interés que no se recogieran habitualmente en los informes del BECAM, los nombrados con más frecuencia son de nuevo la tuberculosis, el SIDA y la meningitis. Por grandes temas, las enfermedades transmisibles son las que generan mayor interés
Tabla 2

\begin{tabular}{|c|c|c|}
\hline INFORMES & $\begin{array}{l}\text { Porcentaje } \\
\text { (y número) de } \\
\text { entrevistados } \\
\text { que han leido } \\
\text { el informe }\end{array}$ & $\begin{array}{c}\text { Porcentaje } \\
\text { (y número) de } \\
\text { los entrevistados } \\
\text { que habiéndolo } \\
\text { leido, lo conside- } \\
\text { ran interesante }\end{array}$ \\
\hline $\begin{array}{l}\text { Morbilidad por enferme- } \\
\text { dades de declaración obli- } \\
\text { gatoria, } 1998\end{array}$ & $\begin{array}{l}31,9 \% \\
(\mathrm{n}=87)\end{array}$ & $\begin{array}{l}91,9 \% \\
(\mathrm{n}=80)\end{array}$ \\
\hline $\begin{array}{l}\text { Hábitos de salud en la po- } \\
\text { blación adulta, } 1998\end{array}$ & $\begin{array}{l}30,0 \% \\
(\mathrm{n}=82)\end{array}$ & $\begin{array}{c}92,7 \\
(\mathrm{n}=76)\end{array}$ \\
\hline $\begin{array}{l}\text { Vigilancia del SIDA/VIH } \\
\text { (datos actualizados a } 30 \\
\text { de marzo de 1999) }\end{array}$ & $\begin{array}{c}50,9 \% \\
(\mathrm{n}=139)\end{array}$ & $\begin{array}{c}97,8 \% \\
(\mathrm{n}=136)\end{array}$ \\
\hline $\begin{array}{l}\text { Prevalencia de afectación } \\
\text { renal en población con } \\
\text { diabetes mellitus Tipo II }\end{array}$ & $\begin{array}{l}27,5 \% \\
(\mathrm{n}=75)\end{array}$ & $\begin{array}{l}90,7 \% \\
(\mathrm{n}=68)\end{array}$ \\
\hline $\begin{array}{l}\text { Impacto de la vacunación } \\
\text { en la enfermedad menin- } \\
\text { gocócica serogrupo C }\end{array}$ & $\begin{array}{c}74,7 \% \\
(\mathrm{n}=204)\end{array}$ & $\begin{array}{c}98,0 \% \\
(\mathrm{n}=200)\end{array}$ \\
\hline $\begin{array}{l}\text { Registro regional de casos } \\
\text { de tuberculosis, } 1998\end{array}$ & $\begin{array}{c}69,3 \% \\
(\mathrm{n}=189)\end{array}$ & $\begin{array}{c}97,3 \% \\
(\mathrm{n}=184)\end{array}$ \\
\hline
\end{tabular}

\section{DISCUSIÓN}

El Boletín Epidemiológico es una publicación que aunque no es conocida ampliamente entre los profesionales médicos atención primaria de la Comunidad de Madrid (el 91,5\% de ellos lo lee alguna vez y el $29,8 \%$ lo hace habitualmente), cuando se lee es bien valorada. Al contactar telefónicamente con el centro, se solicitaba que se pusiesen al teléfono o bien los médicos que más cerca se encontraban de la recepción en ese momento, o bien aquellos que estuviesen disponibles; este hecho pudiera haber introducido sesgos al pensar que dichos médicos fueran diferentes al conjunto de la población; y es difícil poder valorar dicho sesgo y/o en qué sentido pudiera interferir en los resultados.

Las opiniones sobre los informes incluidos en el mismo entre aquellos que los han leído son, en general, muy favorables, con- 
siderándolo más de $90 \%$ interesante o muy interesantes. Ante estos resultados, se puede pensar que ha mejorado considerablemente la imagen de esta publicación entre sus principales destinatarios. Los temas que suscitan más interés, en líneas generales, son las enfermedades transmisibles y dentro de ellas la tuberculosis, la meningitis y la infección VIH/Sida. Parece que los informes relacionados con un problema de salud concreto provocan mayor expectación, así como los relacionados con enfermedades que generan una intervención.

El hecho de que la vigilancia epidemiológica de las enfermedades crónicas se haya iniciado en años recientes, puede hacer que no se asuma todavía como un tema propio de un boletín epidemiológico, por lo que tienen menor interés y se sugieren menos como temas propuestos por los entrevistados.

En cuanto a la utilidad que supone la información publicada para el desempeño de su trabajo (media de 3,5), coincide con lo descrito en otros estudios que evalúan la pertinencia y ayuda de la información contenida en boletines similares al BECAM, tanto en la práctica asistencial como en la actualización de los temas ${ }^{2,3}$. En concreto una encuesta realizada por Gordon $\mathrm{MD}^{4}$, la cual evaluaba un boletín de devolución de la información a médicos, encontró que lo consideraban como un complemento y suplemento al resto de lecturas relacionadas con su profesión. Aún así el número de estudios de estas características es muy limitado, por lo que nos es imposible poder establecer comparaciones más amplias.

Aunque la percepción que los profesionales de atención primaria tienen del BECAM es bastante favorable, se requeriría un nuevo estudio cualitativo diseñado específicamente para conocer en profundidad los intereses y expectativas de los médicos de primaria, tanto en los temas como en el enfoque de los mismos.

Si pensamos en la mejor forma de alcanzar el acceso a la información desde cualquier punto de la geografía mundial de una manera rápida, fácil y cómoda, hemos de centrarnos en las potencialidades que ofrece hoy en día internet. Cabe destacar en este sentido que desde julio de 2001 el BECAM ya figura en formato electrónico en la página web de la Consejería de Sanidad de la Comunidad de Madrid, permitiendo a sus lectores la suscripción al mismo. Este hecho facilita poner a disposición de cualquier persona toda la información generada por los sistemas de vigilancia epidemiológica de una manera más oportuna, que siguiendo los cauces y plazos de tiempo que exigen su edición a través de una imprenta. Aún así es muy pronto para poder valorar el impacto que esta nueva forma de presentación puede tener entre los lectores del BECAM y el resto de usuarios de internet, y junto con la investigación cualitativa mencionada en el párrafo anterior, esta valoración del BECAM electrónico deberá formar parte de estudios futuros.

\section{AGRADECIMIENTOS}

Queremos agradecer la colaboración de todos los médicos participantes en este estudio, haciendo extensible nuestro agradecimiento a todos aquellos profesionales que actúan como notificadores a los diferentes Sistemas de Vigilancia de la Comunidad de Madrid, ya que sin ellos la publicación de este Boletín Epidemiológico no sería posible.

\section{BIBLIOGRAFÍA}

1. Marrugat de la Iglesia J. Encuesta de opinión de los lectores de la Revista Española de Cardiología. Rev Esp Cardiol 1997; 50: 1-4.

2. Hutchinson KA, Cotter Sm, Dollman WB QE-Mail-improving comunication between the hospital and general practitioners. Aust Fam Physician. 1997 Jul; 26 Suppl 2:S99-103.

3. Rea L, Cronk S, Conly J. An infection control bulletin as an educational tool: is it useful? Can J Infect Control 1992. Summer; 7 (2):45-7.

4. Gordon MD. Helping general practitioners to Set up with the literature: evaluation of an RCGP initiativa. Med Educ 1984. May; 18(3): 174-7.

Rev Esp Salud Pública 2002, Vol. 76, N. ${ }^{\circ}$ 


\section{ANEXO 1}

Cuestionario para la evaluación de la distribución y los contenidos Boletín Epidemiológico de la Comunidad de Madrid

Datos personales a rellenar antes de llamar

1. Fecha de la entrevista:

2. Centro de Trabajo

3. Teléfono

4. Puesto de Trabajo: (Coordinador/No coordinador de EAP)

\section{INTRODUCCIÓN}

- Buenos días, soy (nombre de la persona que efectúa la llamada). Le llamo desde el Servicio de Epidemiología de la Comunidad de Madrid. Estamos llevando a cabo una encuesta para valorar la distribución y el interés de los contenidos del Boletín Epidemiológico ?Sería Vd. tan amable de avisar a un médico?

\section{Viene el médico}

- Buenos días, soy (nombre de la persona que efectúa la llamada). Le llamo desde el Servicio de Epidemiología de la Comunidad de Madrid. Estamos llevando a cabo una encuesta para valorar la distribución y el interés de los contenidos del Boletín Epidemiológico

- ¿Será Vd. tan amable de dedicar unos minutos a responder a unas preguntas sobre el mismo?

$\square$ SI $\square$ NO

\section{CUESTIONARIO}

P1. ¿Me puede Vd. decir cual es su puesto de trabajo?

$\square$ Médico de familia: $\square$ de equipo

$\square$ de cupo
$\square$ pediatra de equipo
$\square$ de cupo

$\square$ Coordinador de Equipo

P2. ¿Conoce Vd. el Boletín Epidemiológico de la Comunidad de Madrid?

$\square$ SI $\square$ NO 
ANEXO 1 (continuación)

Cuestionario para la evaluación de la distribución y los contenidos Boletín Epidemiológico de la Comunidad de Madrid

(Explicar: publicación bimensual que edita el Servicio de Epidemiología de la Comunidad de Madrid con temas de Salud Publica. Hasta el año pasado su portada era gris, y en el 2000 ha pasado a ser morada; figurando en su contraportada el mapa de la áreas sanitarias de la Comunidad de Madrid)

SI $\square$ NO $\rightarrow$ fin de entrevista

P3. En el último año ¿cuántos de los Boletines Epidemiológicos publicados ha visto Vd aproximadamente?

$\square 1 \square 2 \square 3 \square 4 \square 5 \square 6$ Otros:_

Dónde los ha visto $\mathrm{Vd}$.

$\square$ En su mesa de trabajo $\square$ En la biblioteca del Centro $\square$ En su domicilio particular

$\square$ En otro lugar:

P4. ¿Sabe que si lo solicita puede recibir el Boletín de forma nominal?

SI $\square$ NO

P5. ¿Lee Vd. alguna vez el Boletín Epidemiológico?

$\mathrm{SI} \rightarrow \mathrm{P} 6$

$\square$ No, no lo he leído nunca

$\square$ Por que razón?

No me parece interesante $\rightarrow \mathrm{P} 11$

No tengo tiempo (fin entrevista)

Otras razones (especificar)

(fin de entrevista)

P6. ¿Con qué frecuencia?

$\square$ Habitualmente $\square$ Esporádicamente $\square$ Casi nunca

P7. ¿Cómo valoraría la información publicada en el Boletín Epidemiológico?

$\square$ Muy interesante $\square$ Interesante $\square$ Poco interesante $\square$ Nada interesante

P8. Puntúe de 1 a 5 la utilidad que tiene la información contenida en el Boletín Epidemiológico para su trabajo. (Siendo 1 ninguna y 5 mucha)

$\square 1 \square 2 \square 3 \square 4 \square 5 \quad$ No sabe: 


\section{ANEXO 1 (continuación)}

Cuestionario para la evaluación de la distribución y los contenidos Boletín Epidemiológico de la Comunidad de Madrid

P9. Como Vd. ya sabe el Boletín Epidemiológico incluye un apartado de contenidos fijos, indique el nivel de interés que le merece cada uno de ellos

\begin{tabular}{|l|c|l|l|l|}
\hline & $\begin{array}{c}\text { muy } \\
\text { interesante }\end{array}$ & interesante & $\begin{array}{c}\text { poco } \\
\text { interesante }\end{array}$ & $\begin{array}{c}\text { nada } \\
\text { interesante }\end{array}$ \\
\hline Enfermedades de Declaración Obligatoria & & & & \\
\hline Brotes Epidémicos & & & & \\
\hline Red de Médico Centinela & & & & \\
\hline Sistema de Notificación Microbiológica & & & & \\
\hline
\end{tabular}

P10. Le voy a listar a continuación los informes sobre temas específicos publicados en el último año, ¿puede señalar los informes que ha leído y si le resultaron interesantes?

A) Morbilidad por Enfermedades de Declaración Obligatoria:

— Lo ha leído
SI
$\square$ NO

— Le ha parecido interesante

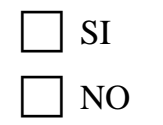

$\square$ ¿Por qué?

$\square$ No le interesa el Tema

$\square$ No le interesa el enfoque que se le ha dado

Otras razones

B) Hábitos de Salud en la Población Adulta de la Comunidad de Madrid

— Lo ha leído

SI

$\square$ NO

— Le ha parecido interesante
SI
$\square$ NO
¿Por qué?
$\square$ No le interesa el Tema
No le interesa el enfoque que se le ha dado
Otras razones 


\section{ANEXO 1 (continuación)}

Cuestionario para la evaluación de la distribución y los contenidos Boletín Epidemiológico de la Comunidad de Madrid

C) Vigilancia del SIDA/VIH en la Comunidad de Madrid

- Lo ha leído

$\square$ SI $\square$ NO

— Le ha parecido interesante

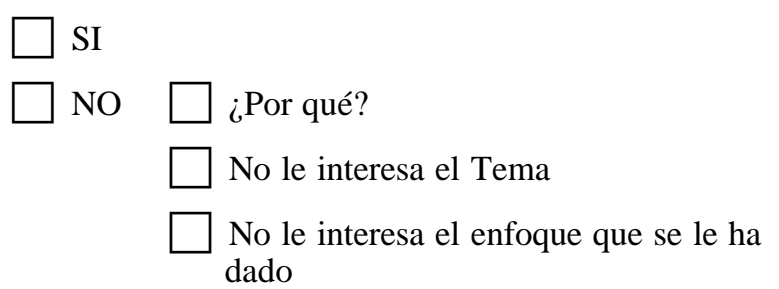
dado

Otras razones

D) Prevalencia de Afectación Renal en Población con Diabetes Mellitus tipo II

- Lo ha leído

$\square$ SI $\square$ NO

— Le ha parecido interesante

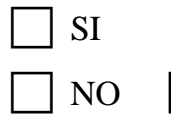

¿Por qué?

$\square$ No le interesa el Tema

$\square$ No le interesa el enfoque que se le ha dado

$\square$ Otras razones

E) Impacto de la Vacunación en la Enfermedad Meningocócica Serogrupo C

— Lo ha leído

$\square$ SI $\square$ NO

— Le ha parecido interesante

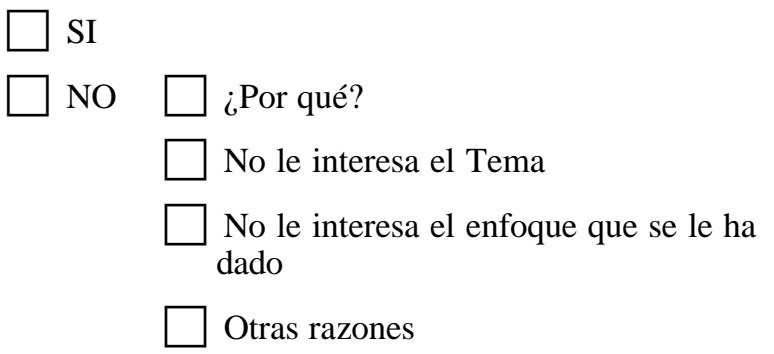




\section{ANEXO 1 (continuación)}

\section{Cuestionario para la evaluación de la distribución} y los contenidos Boletín Epidemiológico de la Comunidad de Madrid

F) Registro de Casos de Tuberculosis en la Comunidad de Madrid

— Lo ha leído
SI
$\square \mathrm{NO}$

— Le ha parecido interesante

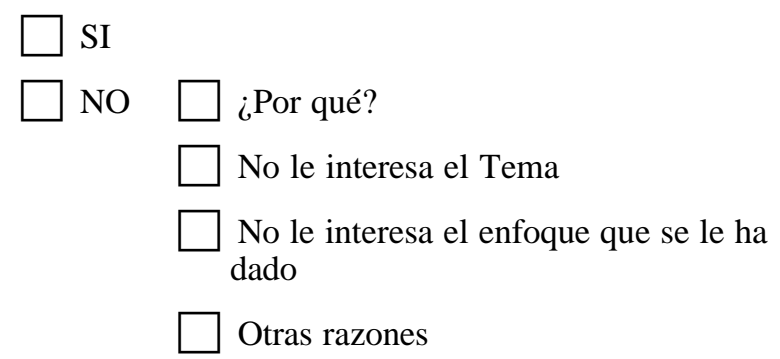

P11. ¿Podría nombrarme cinco temas que le resulten de interés para ser incluidos en el Boletín epidemiológico, por orden de interés?

$$
\begin{aligned}
& 1 \\
& 2 \\
& 3 \\
& 4
\end{aligned}
$$$$
3
$$$$
5
$$ 